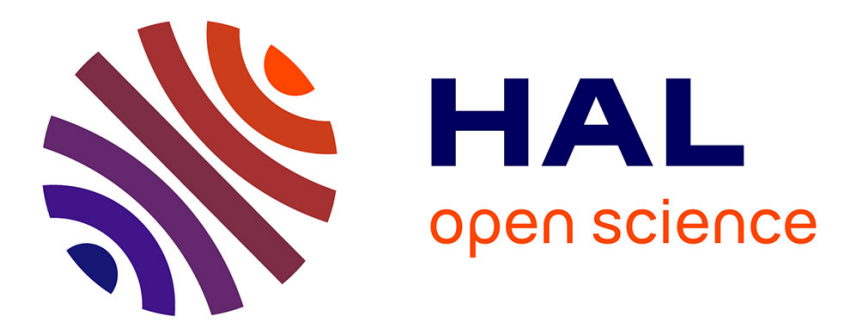

\title{
Wrist Motion Recognition by Using Electromyographic Signals
}

Jing Luo, Chenguang Yang, Chao Liu, Yuxia Yuan, Zhijun Li

\section{To cite this version:}

Jing Luo, Chenguang Yang, Chao Liu, Yuxia Yuan, Zhijun Li. Wrist Motion Recognition by Using Electromyographic Signals. ICARM 2019 - 4th IEEE International Conference on Advanced Robotics and Mechatronics, Jul 2019, Toyonaka, Japan. pp.130-135, 10.1109/ICARM.2019.8833665 . lirmm02315573

\section{HAL Id: lirmm-02315573 https://hal-lirmm.ccsd.cnrs.fr/lirmm-02315573}

Submitted on 14 Oct 2019

HAL is a multi-disciplinary open access archive for the deposit and dissemination of scientific research documents, whether they are published or not. The documents may come from teaching and research institutions in France or abroad, or from public or private research centers.
L'archive ouverte pluridisciplinaire HAL, est destinée au dépôt et à la diffusion de documents scientifiques de niveau recherche, publiés ou non, émanant des établissements d'enseignement et de recherche français ou étrangers, des laboratoires publics ou privés. 


\title{
Wrist Motion Recognition by Using Electromyographic Signals
}

\author{
Jing Luo ${ }^{1}$, Chenguang Yang ${ }^{2, *}$, Chao Liu ${ }^{3}$, Yuxia Yuan ${ }^{4}$, Zhijun $\mathrm{Li}^{5}$
}

\begin{abstract}
Wrist motion classification is a very common research topic in scientific study. However, wrist motion recognition of the surgeon is often neglected in the robot-assisted surgery or surgical training. Therefore, the objective is to develop a classification method to recognize wrist motion of the surgeon. In order to do that, we present a linear discriminant analysis (LDA) algorithm involving surface electromyography (sEMG) signals to evaluate the motions in this paper. Firstly, sEMG signals are collected by using a MYO armband which can be worn on the forearm of a subject. Root-mean-square (RMS) and waveform length (WL) feature are extracted from the sEMG signals and then those features are regarded as input of the LDA to train the classifier. As a result, we can obtain a classifier to recognize four kinds of wrist motions. Classification experiment is performed by two subjects. The experimental results have been demonstrated by using the proposed approach and it is shown that the accuracy of wrist motion by using RMS feature is higher than that of by using WL feature.
\end{abstract}

\section{INTRODUCTION}

Recently, motion capture technology (MCT) is very widely used in many areas involving 3D digital animation, sports and exercise research, medical applications, and neuroscience research, etc. [1] [2] [3] [4]. The motion capture covers position tracking of human, feature extraction, pattern and recognition [5].

In order to recognize motion pattern, kinematic information, such as position, angle, velocity, can be used as input of MCT. At the same time, physiological signal also can be applied to evaluate the motion pattern of human [6] [7] [8]. In general, it cannot acquire neural information of human motion pattern according to the physical information. However, the physiological signals, such as surface electromyogram (sEMG), electroencephalogram(EEG), and electrooculogram (EOG), can help human to analyse neural pattern of motion [9] [10]. The sEMG signals indicate the effect of human's motor unit action potentials of the muscle fiber. Motion

This work was partially supported by National Nature Science Foundation (NSFC) under Grants 61861136009 and 61811530281, CNRS-NSFC Grants PRC2104, and LabEx NUMEV Grant.

${ }^{1}$ J. Luo is with the Key Laboratory of Autonomous Systems and Networked Control, School of Automation Science and Engineering, South China University of Technology, Guangzhou, 510640, China. He was also with Department of Robotics, LIRMM, UMR5506, University of MontpellierCNRS, 161 rue Ada, 34095 Montpellier, France.

${ }^{2} \mathrm{C}$. Yang is with the Bristol Robotics Laboratory, University of the West of England, Bristol, BS16 1QY. U.K.

${ }^{3} \mathrm{C}$. Liu is with Department of Robotics, LIRMM, UMR5506, University of Montpellier-CNRS, 161 rue Ada, 34095 Montpellier, France.

${ }^{4}$ Y. Yuan is with the School of Automation Science and Engineering, South China University of Technology, Guangzhou, 510640, China.

${ }^{5} \mathrm{Z}$. Li is is with the Department of Automation, University of Science and Technology of China, Hefei 230026, China.

*Corresponding author. Email: cyang@ieee.org. intention pattern of the human can be evaluated by using sEMG signals [11] [12].

Many achievements indicated that feature of the sEMG signals could further improve the recognition accuracy of the motion pattern [13] [14] [15]. According to [16], Atoufi et al. used mean absolute values (MAV) of the sEMG signals as the feature to evaluate the muscle synergy. Yang et al. used variety of EMG signal variations to recognize multiple finger motions [17]. In [18], a five time series features was presented to compare the performance of motion pattern by using MYO armband. A common spatial pattern (CSP) feature showed a better performance in comparison with time domain (TD) features for classification accuracy [19]. In [20], Akhlaghi et al. used ultrasound imaging of human arm to recognize the complex volitional hand motion in real time. Bhattacharya et al. proposed a hybrid multi-feature method to control limb prosthses for human computer interfaces [21]. A top and slope (TAS) feature of sEMG signals were introduced to detect lower limb human motion and it could provid high accuracy in the experiments [22]. According to [23], Daubechies wavelet transform method was presented to evaluate the performance of the feature extraction.

To improve the recognition accuracy of the motion pattern, many researchers have concentrated on how to analyse the motion intention [24] [25]. Chambon [26] et al. proposed a deep learning architecture with multivariate and multimodal time series for sleep stage classification. In [27], a hybrid algorithm involving Bayesian and neural networks method was developed to motion classification for human-robot interfaces. A fuzzy logic algorithm was presented for wrist movements classification [28]. Ahsan et al. used an optimized neural network to evaluate EMG motion pattern [29]. In [30], a special subject-independent based decoding model was proposed to decode the wrist motions and hand motions.

Inspired by the feature extraction method and motion classification algorithms, a LDA method with different features was developed to recognize the wrist motions of the surgeon. In this work, we used WL and RMS feature as the input of classification model. In the process of experiment, we founded that the RMS feature can be achieved a higher accuracy in comparison with that of the WL feature. The feasibility of the proposed wrist motion recognition method was demonstrated by the experimental results.

The rest paper's structure is as below. The proposed method of data acquisition, feature extraction and classification are presented in Section II. Section III presents the experimental results which introduce the experimental setup and wrist motion classification experiment. Section IV contains the conclusion and the suggestions for future 
research.

\section{Proposed Method}

We propose a wrist motion framework as shown in Fig. 1 in this paper. It can be seen that this framework contains sEMG signals preprocessing, feature extraction, and classifier.

As shown in Figs. 2(a)-(d), four types of wrist motions are studied $^{1}$. In the experiment, the subject is requested to do the wrist motion with maximal angle.

\section{A. Data acquisition and feature extraction}

As shown in Fig. 1, MYO armband (Thalmic Labs Inc.) is mainly utilized for acquisition of sEMG signals and it wears on the forearm of the subject ${ }^{2}$. The collected sEMG signal is showed in Fig. 3.

Root-mean-square (RMS) and waveform length (WL) are utilized to describe the features of the collected sEMG signals.

RMS feature of the sEMG signals is defined as below:

$$
F_{R M S}=\sqrt{\frac{1}{W_{r m s}} \sum_{i=1}^{W_{r m s}} n_{i}^{2}}
$$

where $W_{r m s}$ represents the length of sampling moving window for RMS. $n_{i}$ represents the collected sEMG signals. $F_{R M S}$ represents the feature of sEMG signals for RMS. Fig. 4 shows the RMS feature.

WL feature of the sEMG signals is defined as below:

$$
F_{W L}=\frac{1}{W_{w l}} \sum_{i=1}^{W_{w l}} \Delta n_{i} .
$$

with

$$
\Delta n_{i}=n_{i}-n_{i-1}
$$

where $W_{w l}$ represents the length of sampling moving window for WL. $n_{i}$ represents the collected sEMG signals. $F_{W L}$ represents the feature of sEMG signals for WL. The WL feature is presented in Fig. 5.

\section{B. Training and classification}

In the training and classification phase, we present a linear discriminant analysis (LDA) to train the classifier to recognize the wrist motion based on RMS feature and WL feature. In the experiment, the subjects train each wrist motion with two minutes, and then repeat 3 times.

In this work, $K_{i}$ is the recognition result of the wrist motion. $F$ is the feature of the collected sEMG signals ${ }^{3}$.

\footnotetext{
${ }^{1}$ The four wrist motion can be described as: wrist to the right (WtR), wrist to the left (WtL), wrist extension (WE), and wrist flexion (WF).

${ }^{2}$ The MYO armband collects the raw sEMG signals by using eight detection electrodes and a nine-axis inertial measurement unit with two hundreds sampling Hertz.

${ }^{3}$ In this paper, the features of the collected sEMG signals contain $F_{R M S}$ and $F_{W L}$.
}

According to the features, we can recognize the wrist motion type.

Based on [31] [32], the wrist motion result $K_{i}$ can be defined as below:

$$
p\left(K_{i} \mid F\right)=\frac{p\left(K_{i}\right) p\left(F \mid K_{i}\right)}{p(F)}
$$

where $p\left(K_{i}\right)$ represents the prior probability of the wrist motion. $p\left(K_{i} \mid F\right)$ represents the posterior probability of the wrist motion by using the sEMG signal's features $F$. When the value of posterior probability is maximum for a certain wrist motion, we can be sure the recognition result by using the LDA method.

In general, $p\left(F \mid K_{i}\right)$ represents the contingent probability of the wrist motion, and it can be defined as below ${ }^{4}$ :

$$
\begin{aligned}
p\left(F \mid K_{i}\right)= & \frac{1}{\sqrt{(2 \pi)^{M} \operatorname{det}(D)}} \\
& \quad \exp \left\{-\frac{1}{2}\left(F-\mu_{i}\right)^{T} D^{-1}\left(F-\mu_{i}\right)\right\}
\end{aligned}
$$

where $M$ represents the number of sEMG signal's feature vector. $\mu_{i}$ represents the mean vector of the wrist motion result $K_{i}$. $D$ represent the covariance matrix for four types of wrist motions.

Logarithm fetch on Eq. 5, we can obtain the maximum value of the $p\left(K_{i} \mid F\right)$. And then the linear discriminant function (LDF) is defined as below:

$$
\delta(k)=F^{T} \mu_{g}+c_{g}
$$

with

$$
\begin{gathered}
\mu_{g}=D^{-1} \mu_{k} \\
c_{g}=-\frac{1}{2} \mu_{k}^{T} D^{-1} \mu_{k}
\end{gathered}
$$

We maximize the LDF $\delta(k)$, the recognition result can be obtained according to the feature vector.

\section{EXPERIMENTS AND RESULTS}

In this section, an experiment is performed to recognize the four kinds of wrist motions (WR, WL, WE, and WF).

The process of this experiment can be presented as in Fig. 6.

\section{A. Experimental setup}

The experimental setup is conducted to evaluate the effectiveness of the wrist motion recognition method.

- Hardware configuration. Hardware configuration contains the MYO armband with 8 sensor $(200 \mathrm{~Hz})$, a work station with i7-3770T CPU $(2.50 \mathrm{GHz})$ and $12 \mathrm{~GB}$ internal storage.

- Software configuration. Software configuration includes MATLAB R2016a, VS 2013, and Windows seven operation system.

${ }^{4}$ In this paper, $p\left(f_{R M S} \mid c_{k}\right)$ can satisfy the multivariate probability distribution (MPD). 


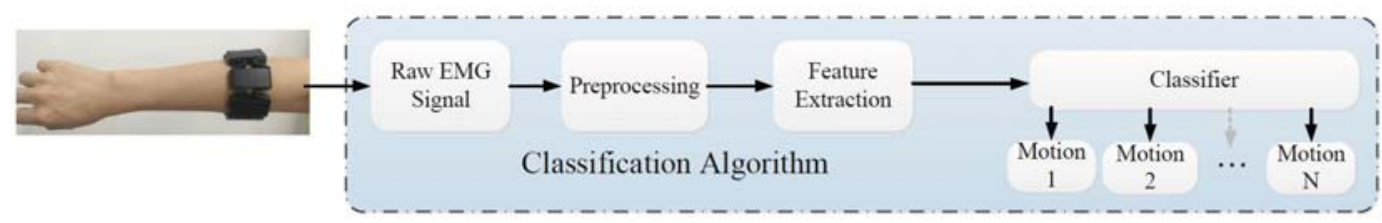

Fig. 1. The wrist motion framework.

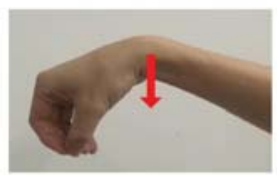

(a) WF

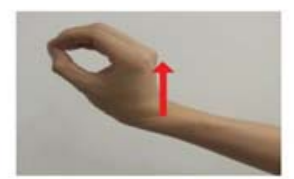

(b) WE

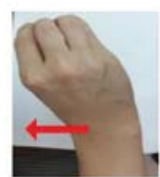

(c) WL

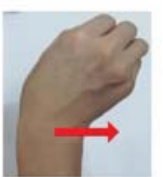

(d) WR

Fig. 2. Four kinds of wrist motions and the placement of the MYO armband on a right forearm.
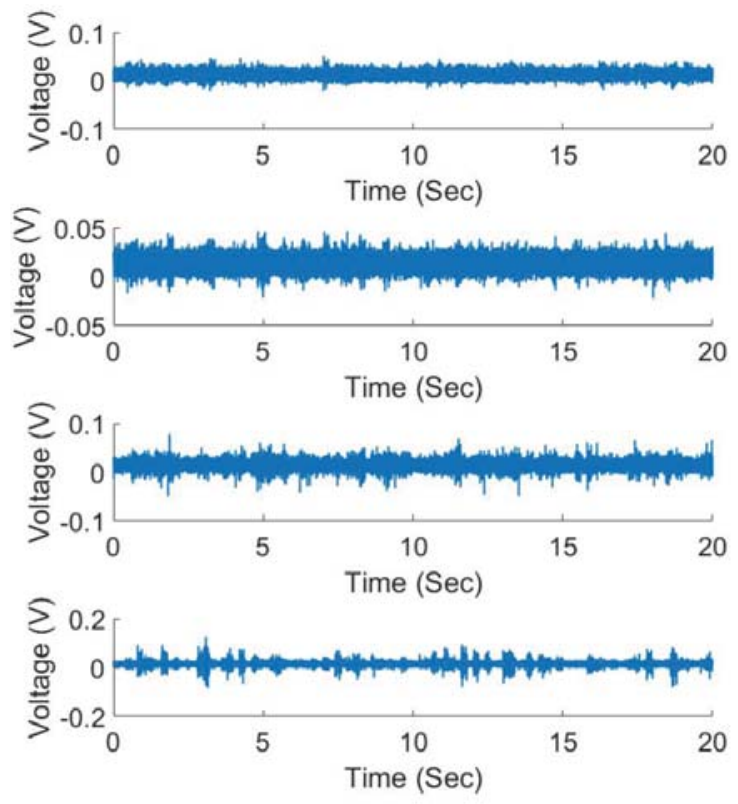

Fig. 3. The collected sEMG signals by using a MYO armband.

Accuracy (ACC) is used to evaluate the feasibility of the proposed method.

In this experiment, two healthy subjects (age 22-30 years old, 2 males) are invited to perform the four types or wrist motion with maximal angle. To minimize the influence of muscle fatigue for the subject, we request each subject perform two times with two minutes for each wrist motion, and then have a rest with thirty minutes. In order to discuss the impact of the difference features, we utilize the $F_{R M S}$ and $F_{W L}$ to represent the sEMG signal's feature. In the pilot experiment, the moving window size of $F_{R M S}$ and $F_{W L}$ are set as $W_{r m s}=W_{w l}=50$. We perform two experiments in this work. The experimental parameters of the two experiments can be presented as:
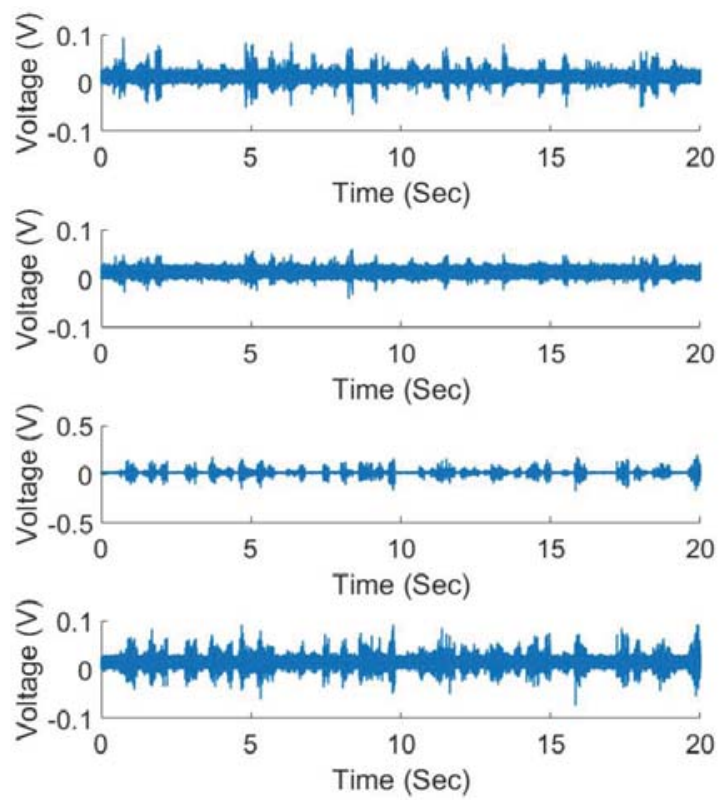

TABLE I

THE EXPERIMENTAL PARAMETERS OF THE TWO EXPERIMENTS

\begin{tabular}{|c|c|c|}
\hline Experiment & Experiment 1 & Experiment 2 \\
\hline Size of training set & $250 * 8$ & $20000 * 8$ \\
\hline Size of testing set & $200 * 8$ & $20000 * 8$ \\
\hline Feature & $F_{R M S}, F_{W L}$ & $F_{R M S}, F_{W L}$ \\
\hline Size of moving window & 50 & 50 \\
\hline
\end{tabular}

\section{B. Wrist motion classification experiment}

In experiment 1 , the classification results are presented in Tables II-III and Fig. 7. In Table II, WtL and WtR can be obtained the best performance of classification in comparison with that of other two wrist motions based on RMS feature. While, in Table III, the performance of classification of WtL 

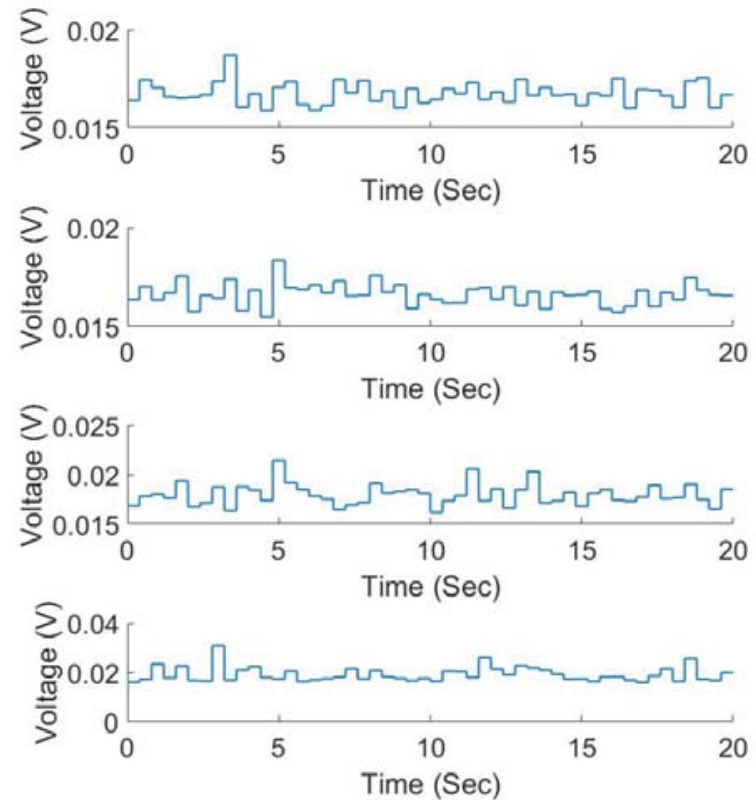

Fig. 4. The feature of sEMG by using RMS.
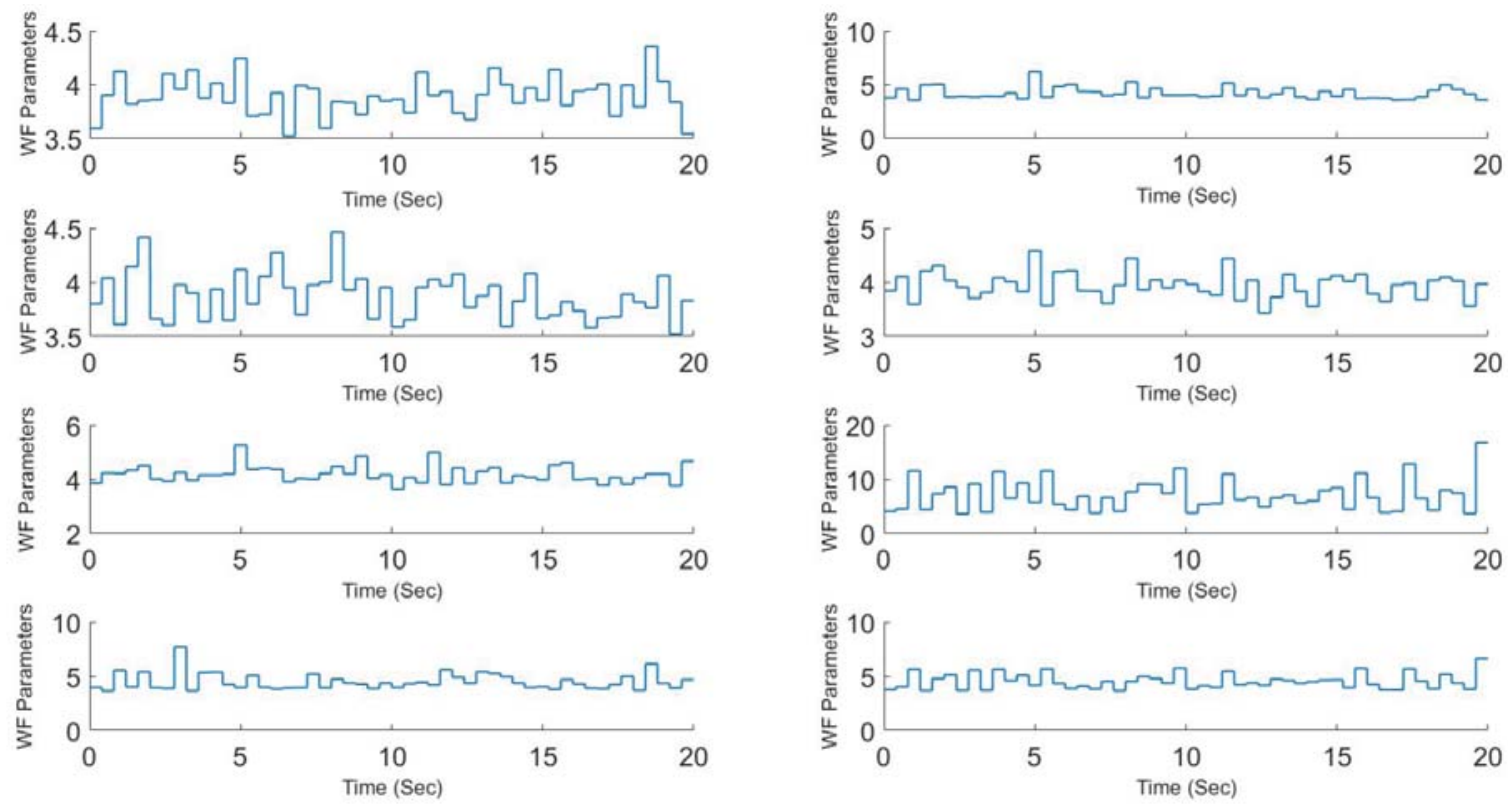

Fig. 5. The feature of sEMG by using WL.

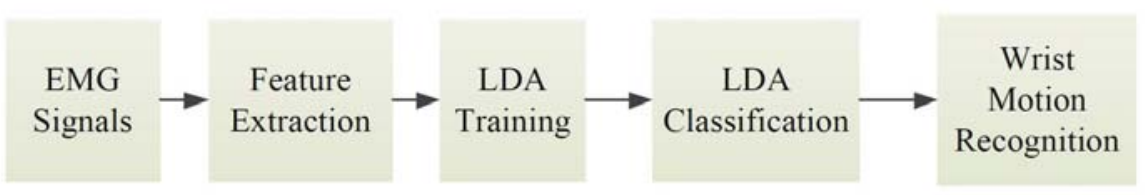

Fig. 6. Experiment process of the proposed wrist gesture classification and wrist stiffness estimation.

is best among four types of wrist motions. It can be concluded WtR,WE and WF) for the same subject. In addition, sEMG that the signal strength is different for wrist motions (WtL, signal for WtL and WtR is relatively strong in experiment 1. 
The average classification accuracy by using the RMS feature is higher than that by using the WL feature from Fig. 7.

TABLE II

THE RESULTS OF WRIST GESTURE CLASSIFICATION FOR EXPERIMENT 1 BY USING RMS FEATURE.

\begin{tabular}{|c|c|c|c|c|}
\hline Gesture & WtL & WtR & WE & WF \\
\hline WtL & 1 & 0 & 0 & 0 \\
\hline WtR & 0 & 1 & 0 & 0 \\
\hline WE & 0 & 0 & 0.8 & 0 \\
\hline WF & 0 & 0 & 0 & 0.7 \\
\hline
\end{tabular}

TABLE III

THE RESULTS OF WRIST GESTURE CLASSIFICATION FOR EXPERIMENT 1 BY USING WL FEATURE.

\begin{tabular}{|c|c|c|c|c|}
\hline Gesture & WtL & WtR & WE & WF \\
\hline WtL & 1 & 0 & 0 & 0 \\
\hline WtR & 0 & 0.7 & 0 & 0 \\
\hline WE & 0 & 0 & 0.8 & 0 \\
\hline WF & 0 & 0 & 0 & 0.8 \\
\hline
\end{tabular}

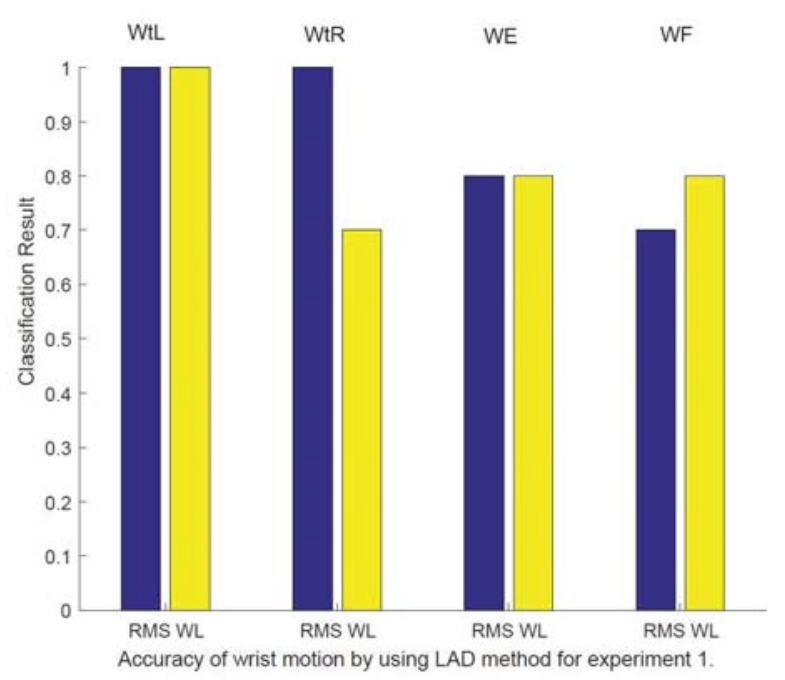

Fig. 7. Accuracy of wrist motion by using LAD method for experiment 1 .

In experiment 2, Tables IV-V and Fig. 8 show the classification experimental results. In Tables IV and $\mathrm{V}$, the performance of classification for WF is best in comparison with WfL, WtR, and WE by using RMS or WL feature. That is to say, sEMG signal for WF is relatively strong.

Compared with the performance between Fig. 7 and Fig. 8, accuracy of classification is higher by using RMS feature for four kinds of wrist motions. What is more, sEMG signal of the subjects vary from person to person. the variability of sEMG signals represent the subjects operational characteristics. According to the experimental results from experiment 1 and 2, it can be demonstrated the wrist motions can be recognized with higher accuracy by using the RMS feature.
TABLE IV

THE RESULTS OF WRIST GESTURE CLASSIFICATION FOR EXPERIMENT 2 BY USING RMS FEATURE.

\begin{tabular}{|c|c|c|c|c|}
\hline Gesture & WtL & WtR & WE & WF \\
\hline WtL & 0.895 & 0 & 0 & 0 \\
\hline WtR & 0 & 0.8 & 0 & 0 \\
\hline WE & 0 & 0 & 0.85 & 0 \\
\hline WF & 0 & 0 & 0 & 1 \\
\hline
\end{tabular}

TABLE V

THE RESULTS OF WRIST GESTURE CLASSIFICATION FOR EXPERIMENT 2 BY USING WL FEATURE.

\begin{tabular}{|c|c|c|c|c|}
\hline Gesture & WtL & WtR & WE & WF \\
\hline WtL & 0.88 & 0 & 0 & 0 \\
\hline WtR & 0 & 0.75 & 0 & 0 \\
\hline WE & 0 & 0 & 0.8 & 0 \\
\hline WF & 0 & 0 & 0 & 1 \\
\hline
\end{tabular}

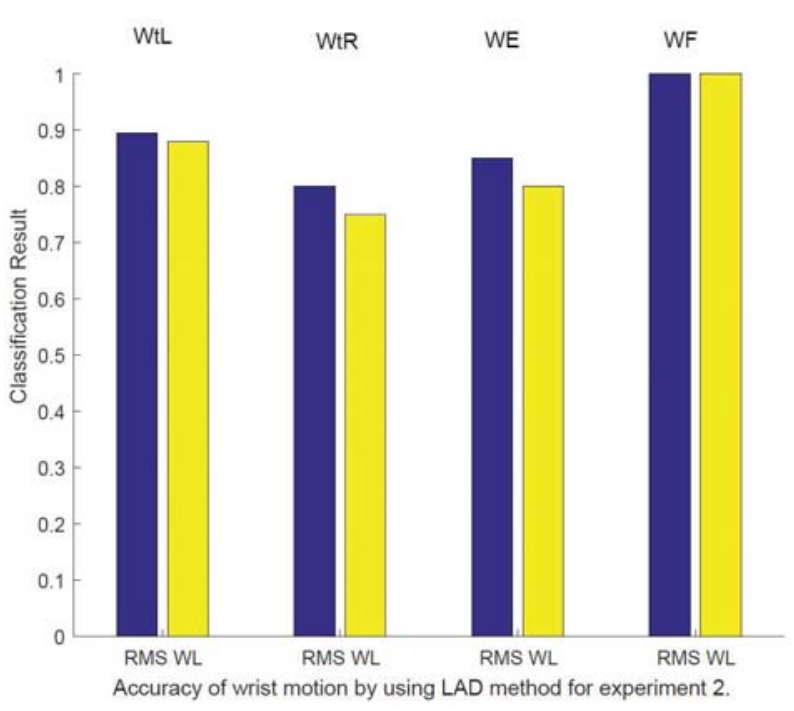

Fig. 8. Accuracy of wrist motion by using LAD method for experiment 2 .

\section{CONCLUSiOn AND FUtURE WORK}

A method based on sEMG signals is developed in this paper to recognize wrist motion of the surgeon. The classification method recognizes wrist motions by using RMS feature and WL feature. Compared with the WL feature, the accuracy of classification is higher when RMS feature are used as the input of LDA classifier. In the experimental results, it is showed that the proposed LDA approach can recognize the wrist motions successfully.

In the future work, several issues should be resolved. First, the different algorithms of feature extraction can reflect the different modes for the sEMG signals. The appropriate combination of feature extraction algorithms can effectively improve the classification accuracy of the motions. Therefore, integration of different feature extraction algorithms for the motion classification should be taken into consideration. 


\section{REFERENCES}

[1] M. Castresana and F. Siles, "Goniometry-based glitch-correction algorithm for optical motion capture data," in 2018 IEEE International Work Conference on Bioinspired Intelligence (IWOBI), pp. 1-8, IEEE, 2018

[2] L. H. Smith and L. J. Hargrove, "Comparison of surface and intramuscular emg pattern recognition for simultaneous wrist/hand motion classification," in Conference proceedings.... Annual International Conference of the IEEE Engineering in Medicine and Biology Society. IEEE Engineering in Medicine and Biology Society. Conference, vol. 2013, p. 4223, NIH Public Access, 2013.

[3] S. Yokota, H. Hashimoto, Y. Ohyama, J.-H. She, D. Chugo, and H. Kobayashi, "Classification of body motion for human body motion interface," in Human System Interactions (HSI), 2010 3rd Conference on, pp. 734-738, IEEE, 2010.

[4] V. Kehri, R. Ingle, S. Patil, and R. Awale, "Analysis of facial emg signal for emotion recognition using wavelet packet transform and svm," in Machine Intelligence and Signal Analysis, pp. 247-257, Springer, 2019.

[5] J. Luo, C. Yang, Q. Li, and M. Wang, "A task learning mechanism for the telerobots," International Journal of Humanoid Robotics, 2019.

[6] Z. Ju and H. Liu, "Human hand motion analysis with multisensory information," IEEE/ASME Transactions on Mechatronics, vol. 19 no. 2, pp. 456-466, 2014.

[7] Z. Ju and H. Liu, "A unified fuzzy framework for human-hand motion recognition," IEEE Transactions on Fuzzy Systems, vol. 19, no. 5 , pp. 901-913, 2011.

[8] H. Liu, Z. Ju, X. Ji, C. S. Chan, and M. Khoury, "Fuzzy qualitative trigonometry," in Human Motion Sensing and Recognition, pp. 35-50, Springer, 2017.

[9] C. Yang, J. Luo, Y. Pan, Z. Liu, and C.-Y. Su, "Personalized variable gain control with tremor attenuation for robot teleoperation," IEEE Transactions on Systems, Man, and Cybernetics: Systems, 2017.

[10] C. Yang, J. Luo, C. Liu, M. Li, and S.-L. Dai, "Haptics electromyogrphy perception and learning enhanced intelligence for teleoperated robot," IEEE Transactions on Automation Science and Engineering, 2018.

[11] J. Han, Q. Ding, A. Xiong, and X. Zhao, "A state-space emg model for the estimation of continuous joint movements," IEEE Transactions on Industrial Electronics, vol. 62, no. 7, pp. 4267-4275, 2015.

[12] D. G. Lloyd and T. F. Besier, "An emg-driven musculoskeletal model to estimate muscle forces and knee joint moments in vivo," Journal of biomechanics, vol. 36, no. 6, pp. 765-776, 2003.

[13] J. Luo, C. Yang, N. Wang, and M. Wang, "Enhanced teleoperation performance using hybrid control and virtual fixture," International Journal of Systems Science, vol. 0, no. 0, pp. 1-12, 2019.

[14] S. Balasubramanian, E. Garcia, N. Birbaumer, E. Burdet, and A. Ramos, "Is emg a viable alternative to bci for detecting movement intention in severe stroke?," IEEE Transactions on Biomedical Engineering, 2018.

[15] M. Gardner, R. Vaidyanathan, E. Burdet, and B. C. Khoo, "Motionbased grasp selection: Improving traditional control strategies of myoelectric hand prosthesis," in Rehabilitation Robotics (ICORR), 2015 IEEE International Conference on, pp. 307-312, IEEE, 2015.

[16] B. Atoufi, E. N. Kamavuako, B. Hudgins, and K. Englehart, "Classification of hand and wrist tasks of unknown force levels using muscle synergies," in Engineering in Medicine and Biology Society (EMBC), 2015 37th Annual International Conference of the IEEE, pp. 16631666, IEEE, 2015.

[17] D. Yang, W. Yang, Q. Huang, and H. Liu, "Classification of multiple finger motions during dynamic upper limb movements," IEEE journal of biomedical and health informatics, vol. 21, no. 1, pp. 134-141, 2017.

[18] Z. Arief, I. A. Sulistijono, and R. A. Ardiansyah, "Comparison of five time series emg features extractions using myo armband," in Electronics Symposium (IES), 2015 International, pp. 11-14, IEEE, 2015.

[19] X. Li, P. Fang, L. Tian, and G. Li, "Increasing the robustness against force variation in emg motion classification by common spatial patterns," in Engineering in Medicine and Biology Society $(E M B C)$ 2017 39th Annual International Conference of the IEEE, pp. 406-409, IEEE, 2017.
[20] N. Akhlaghi, C. A. Baker, M. Lahlou, H. Zafar, K. G. Murthy, H. S. Rangwala, J. Kosecka, W. M. Joiner, J. J. Pancrazio, and S. Sikdar, "Real-time classification of hand motions using ultrasound imaging of forearm muscles," IEEE Transactions on Biomedical Engineering, vol. 63, no. 8, pp. 1687-1698, 2016.

[21] A. Bhattacharya, A. Sarkar, and P. Basak, "Time domain multi-feature extraction and classification of human hand movements using surface emg," in Advanced Computing and Communication Systems (ICACCS), 2017 4th International Conference on, pp. 1-5, IEEE, 2017.

[22] J. Ryu, B.-H. Lee, and D.-H. Kim, "semg signal-based lower limb human motion detection using a top and slope feature extraction algorithm,' IEEE Signal Processing Letters, vol. 24, no. 7, pp. 929932, 2017.

[23] I. Elamvazuthi, G. Ling, K. R. K. Nurhanim, P. Vasant, and S. Parasuraman, "Surface electromyography (semg) feature extraction based on daubechies wavelets," in Industrial Electronics and Applications (ICIEA), 2013 8th IEEE Conference on, pp. 1492-1495, IEEE, 2013.

[24] J. He, D. Zhang, X. Sheng, S. Li, and X. Zhu, "Invariant surface emg feature against varying contraction level for myoelectric control based on muscle coordination," IEEE journal of biomedical and health informatics, vol. 19, no. 3, pp. 874-882, 2015.

[25] X. Zhu, J. Liu, D. Zhang, X. Sheng, and N. Jiang, "Cascaded adaptation framework for fast calibration of myoelectric control," IEEE Transactions on Neural Systems and Rehabilitation Engineering, vol. 25, no. 3, pp. 254-264, 2017.

[26] S. Chambon, M. N. Galtier, P. J. Arnal, G. Wainrib, and A. Gramfort, "A deep learning architecture for temporal sleep stage classification using multivariate and multimodal time series," IEEE Transactions on Neural Systems and Rehabilitation Engineering, 2018.

[27] N. Bu, M. Okamoto, and T. Tsuji, "A hybrid motion classification approach for emg-based human-robot interfaces using bayesian and neural networks," IEEE Transactions on Robotics, vol. 25, no. 3, pp. 502-511, 2009

[28] M. Karuna, C. Ganesh, R. P. Das, and M. V. Kumar, "Classification of wrist movements through emg signals with fuzzy logic algorithm," in 2017 International Conference on Energy, Communication, Data Analytics and Soft Computing (ICECDS), pp. 2258-2261, IEEE, 2017.

[29] M. R. Ahsan, M. I. Ibrahimy, and O. O. Khalifa, "Emg motion pattern classification through design and optimization of neural network," in Biomedical Engineering (ICoBE), 2012 International Conference on, pp. 175-179, IEEE, 2012

[30] C. W. Antuvan, S.-C. Yen, and L. Masia, "Simultaneous classification of hand and wrist motions using myoelectric interface: Beyond subject specificity," in Biomedical Robotics and Biomechatronics (BioRob), 2016 6th IEEE International Conference on, pp. 1129-1134, IEEE, 2016.

[31] J. Lu, K. N. Plataniotis, and A. N. Venetsanopoulos, "Face recognition using LDA-based algorithms.," IEEE Transactions on Neural Networks, vol. 14, no. 1, pp. 195-200, 2003.

[32] P. Jing, D. R. Heisterkamp, and H. K. Dai, "LDA/SVM driven nearest neighbor classification," IEEE Transactions on Neural Networks, vol. 14 , no. 4, pp. 940-2, 2003. 